\title{
A game-theoretic framework for dynamic cyber deception in Internet of Battlefield Things
}

\author{
Ahmed H. Anwar \\ Charles Kamhoua \\ Nandi Leslie \\ a.h.anwar@knights.ucf.edu \\ charles.a.kamhoua.civ@mail.mil \\ nandi.o.leslie.ctr@mail.mil \\ US Army Research Laboratory \\ Adelphi, Maryland
}

\begin{abstract}
Cyber deception techniques are crucial to protect networks in battlefield settings and combat malicious cyber attacks. Cyber deception can effectively disrupt the surveillance process outcome of an adversary. In this paper, we propose a novel approach for cyber deception to protect important nodes and trap the adversary. We present a sequential approach of honeypot placement to defend and protect the network vital nodes. We formulate a stochastic game to study the dynamic interactions between the network administrator and the attacker. The defender makes strategic decisions about where to place honeypots to introduce new vulnerabilities to the network. The attacker's goal is to develop an attack strategy to compromise the nodes of the network by exploiting a set of known vulnerabilities. To consider a practical threat model, we assume that the attacker can only observe a noisy version of the network state. To this end, both players solve a partially observable stochastic game (POSG). Finally, we present a discussion on existing techniques to solve the formulated game and possible approaches to reduce the game complexity as part of our ongoing and future research.
\end{abstract}

\section{CCS CONCEPTS}

- Networks $\rightarrow$ Network security; Mobile and wireless security.

\section{KEYWORDS}

network security, game theory, cyber deception, IoBT.

\section{ACM Reference Format:}

Ahmed H. Anwar, Charles Kamhoua, and Nandi Leslie. 2019. A gametheoretic framework for dynamic cyber deception in Internet of Battlefield Things. In 16th EAI International Conference on Mobile and Ubiquitous Systems: Computing, Networking and Services (MobiQuitous), November 12-14, 2019, Houston, TX, USA. ACM, New York, NY, USA, 5 pages. https://doi.org/10.1145/3360774.3368204

ACM acknowledges that this contribution was authored or co-authored by an employee, contractor, or affiliate of the United States government. As such, the United States government retains a nonexclusive, royalty-free right to publish or reproduce this article, or to allow others to do so, for government purposes only.

MobiQuitous, November 12-14, 2019, Houston, TX, USA

(C) 2019 Association for Computing Machinery.

ACM ISBN 978-1-4503-7283-1/19/11 ..\$15.00

https://doi.org/10.1145/3360774.3368204

\section{INTRODUCTION}

Modern computer networks are highly connected and heterogeneous in order to provide more complicated services and adapt to increasing and rapidly changing demands. For instance, these networks connect computers with different operating systems and protocols, wireless networks accommodate different access technologies and are able to provide services through a Wi-Fi LAN or through a cellular network as in heterogeneous wireless networks. Moreover, an increasing number of devices are being added to networks everyday [16]. For example, the deployment of wirelessenabled devices [e.g., Internet of Things (IoT), robots, sensors] has made networks larger and denser. Hence, networks are prone to higher levels of interference, which makes them more vulnerable. The diversity of devices also makes maintaining them (e.g., patching vulnerabilities) a much more challenging management problem. Such security issues are even more challenging in the military environment.

The IoT network structure is also being deployed in battlefield contexts, where is is known as the Internet of Battlefield Things (IoBT) $[20,21]$. In a broader sense, the IoBT also refers to devices useful for military battles that may communicate over tactical networks other than the Internet. Therefore, protecting the resilience and robustness of critical nodes of such a network in a battlefield is crucial. In this paper, we propose an approach for optimizing cyber deception to prevent possible attackers from characterizing effective attack strategies. It is evident that attackers gather information about the targeted systems/networks before launching their attacks [31]. Information gathering (also called the reconnaissance stage) is the stage of attack in which an attacker collects inside information about the targeted network using a set of tools and scanning techniques [14]. In rare scenarios, attackers are able to obtain intelligence information that describes the internal structure of the targeted network. Attackers typically map the targeted network using software scanning tools like Nmap [25] or traffic analysis. On the other side, the network administrator (the defender) can effectively protect his network at this early stage of reconnaissance by deceiving the attacker and manipulating the network interfaces to disguise the true state of the network. Specifically, we investigate an attack scenario in which the defender protects critical nodes and important system components via introducing false information (i.e., deception) to disrupt the attacker's decision-making, providing him with a false sense of certainty. 
Cyber Deception Games (CDG) are a recent but growing topic in the game theory literature, modeling scenarios in which the defender protects the network by manipulating its state, and in some cases using decoys or deceptive signals [8, 10, 18, 24, 35, 35]. The defender may camouflage nodes to hide critical networked devices. Recent work formulated honeypot deception games as a one-shot game [5, 9, 36]. Schlenker et al. [32] proposed a defense approach based on a Stackelberg game model to increase the attacker uncertainty about the system parameters to misguide the network scanning tools. Hence, the attacker gets false information about what operating system is running on what machine, what port is assigned to which service, and the names of subnetworks and active users. In [23] the authors presented a Stackelberg game model in which the defender chooses optimal mitigations that reduce the capability of the attacker to achieve his goals. The authors also investigated complexities and scalability challenges of such problems.

The applications of these sorts of game theoretic models can shape the understanding of robustness for connected devices in a tactical network (e.g., IoBT). Over the past several decades, there has been a growing concern with network resilience for industries and government, given that security breaches are pervasive, and cyber deception can be an effective proactive network resilience approach to this end. For example, in 2019, the U.S. Department of the Army published a document on its strategy for military deception for multi-domain operations [3], including network security, that addresses deception-this includes the presentation of false weakness in information systems-as a means to dissuade network attacks.

In residential and commercial networks, the attacker experiences a static view of the network state since the defender does not change security policies and settings very frequently. However, in a fast-paced battlefield network, both the defender and the attacker should be aware of the network state dynamically. Therefore, a one-shot game model falls short in studying such a network. To this end, we propose a practical partially observable stochastic game (POSG) model to capture the dynamics of the deception game played between the attacker and the defender.

Despite the fact that game-theoretic defense techniques have been used and implemented for several years now [1, 30, 33] many other questions are still open and more research has to be done. Providing effective defense strategies for any system depends on the accuracy of the game model used to describe the attributes of such a system. Hence, tailoring a game model to capture IoBT characteristics is essential in this case to protect the critical network nodes. In particular, previous work has not capture the type of dynamic interaction under uncertainty and the types of dynamic network reconfiguration strategies that we believe are critical understanding the IoBT case.

Attack graph: Each node of the IoBT network represents an autonomous entity that is self-aware of the surrounding environment as well as its neighboring nodes. An attacker causes more damage to the network by targeting critical nodes and hence the defender needs to protect the network via inserting honeypot nodes that at tract adversaries while securing network critical nodes. Each node runs a set of applications and thus has a set of known vulnerabilities that can be exploited to reach other hosts in the network. If such a node is accessible through another node on the graph, a directed edge connects the two nodes to capture this reachability relation [11]. When the defender adds new honeypots to deceive the adversary, the set of vulnerabilities on the attack graph observed by the attacker dynamically changes.

Previous work on attack graphs assumed that the adversary could obtain perfect information of the network [12, 34] and hence can generate its true attack graph using any of the generation tools [27, 28]. A static version of this attack model in the context of deception games was considered in [8, 29]. Authors in [13] presented a case study of a deception game using honeypot allocation with fully observable Stackelberg game model with promising preliminary results. The authors in [17] proposed a scalable optimal solution technique for zero-sum security games on static graphs.

Partially observable stochastic games: The adversary spends time to explore and map out the network. Knowing the network state, the attacker can maximize her reward by attacking critical nodes via exploiting the discovered vulnerabilities. A value is associated with each node that reflects its importance on the attack graph. In such scenario, the set of vulnerabilities associated with each node, and hence the associated attack graph, varies with time. Therefore, the attacker is uncertain about the true state of the network.

In addition to the attacker having a partial observation of the network, the defender is also uncertain about which subset of vulnerabilities has already been exploited by the attacker. To study this interaction, we formulate a POSG between the network defender and the attacker. The players' observation of the state is asymmetric. In other words, the attacker encounters state uncertainty, while the defender fully observes the network and the associated attack graph but does not perfectly observe which vulnerabilities are being exploited. A related model was investigated in [11] with a different formulation of the game model which led to a lot of computational complexities, and hence, authors used relaxation assumptions to provide an approximate solution. Therefore, a POSG model perfectly fits well to model the considered threat and defense models.

Cyber deception is still an under-studied topic in the literature, and our paper provides a novel framework to tackle the dynamic aspect of deception within a game theoretic perspective. To consider a practical threat model, this paper considers a game where both players partially observe each others strategies. To solve this game we leverage existing techniques and provide new approaches to overcome the curse of dimensionality that is inherent in this class of games. Our contributions can be summarized as follows:

- We propose a novel stochastic game model to investigate and protect a connected graph of targeted nodes against an attacker.

- We consider a practical threat model in which we account for the uncertainties experienced by both the defender and the attacker.

- We develop a partially observable stochastic game, where the defender goal is to stop the attacker early in the Cyber kill chain and prevents the subsequent more dangerous phases.

- We discuss the game solution and highlight approaches to overcome the complexity of the stochastic game using existing algorithms. 


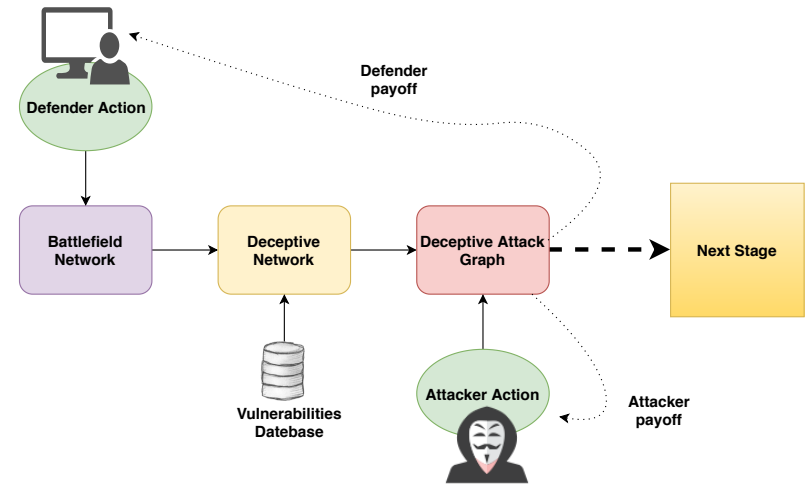

Figure 1: System model diagram shows the relation between network state and actions of both players

The rest of the paper is organized as follows: In Section 2 we describe the system model. In Section 3 we rigorously formulate the game between the two players. Then in Section 4 we discuss feasible approaches to solve the game. We discuss the game complexity and propose several approaches to reduce it. We conclude in Section 5.

\section{SYSTEM MODEL}

We now present in more detail a novel formulation to study deception through honeypot allocation with partial state information. The defender can either place a new honeypot, or disguise a honeypot as a normal host. Consider a network with an arbitrary number of hosts. The defender is aware of the existing risk and that the network nodes are targeted. On the other side, the attacker expects that some of the vulnerabilities are decoy. Therefore, the attacker does not know the true identity of each vulnerability. The identity determines whether the vulnerability belongs to a true node or to a honeypot.

Given the network vulnerabilities and the deception action taken by the defender, an attack graph can be generated based on the node's configuration and the current known vulnerabilities [26] We assume that such vulnerability information is public and can be obtained by both players. Therefore, the attacker is capable of exploiting the available vulnerabilities to reach her goal. Fig.1 illustrates the relation between the network state and the actions of the players. The attack graph is generated based on the true network state and the deceptive actions taken by the defender. The attacker observes the deceptive attack graph which is a noisy version of the exploitable vulnerabilities of the network and hence responds without knowing the true identities of the graph vertices.

The actions taken by the attacker over the vulnerability graph are not random, however they point toward reaching a targeted node. Moreover, the attacker future action space depends on the previous actions. To consider a practical threat model, we assume that the defender has partial observation of the attacker actions. Hence, the defender does not know exactly which nodes have been targeted by the attacker. In the next section we formally define our game model as a POSG between the network defender and the attacker.

\section{PROBLEM FORMULATION}

Let $\mathcal{G}(\mathcal{V}, E)$ represents an attack graph, where $\mathcal{V}$ is a set of nodes and $E$ is a set of all edges. In specific, a directed edge $e_{u, v} \in E$ for some $u, v \in \mathcal{V}$ exists when an attacker can reach host $v$ from host $u$ by exploiting an existing service or vulnerability on the attack graph. A two-player zero-sum partially observable stochastic game is defined as a tuple $(\mathcal{N}, \mathcal{S}, \mathcal{A}, \mathcal{O}, \mathcal{P}, \mathcal{R})$, where,

- $\mathcal{N}=\{1,2\}$ is the set of players, where player 1 is the network defender and player 2 is the attacker

- $\mathcal{S}$ is a finite set of all possible states.

- $\mathcal{A}=\mathcal{A}_{1} \times \mathcal{A}_{2}$ is the game action space, where $\mathcal{A}_{1}$ and $\mathcal{A}_{2}$ is the defender and attacker action spaces, respectively. An action $a_{1}^{t} \in \mathcal{A}_{1}$ played by the defender at time $t$ defines the state of the network. An attacker action $a_{2}^{t} \in \mathcal{A}_{2}$ at time $t$ determines the reward of both players.

- $O=O_{1} \times O_{2}$ is a finite set of observations.

- $\mathcal{P}=\left[p_{i, j}\right]$ is a set of Markovian state and observation transition probabilities. $\operatorname{Pr}\left(s^{j}, o \mid s^{i}, a_{1}, a_{2}\right)$ is the transitional probability to state $s^{j}$ and observation $o$ from state $s^{i}$ under the joint action $\left(a_{1}, a_{2}\right)$.

- $\mathcal{R}=\left\{\mathcal{R}_{1}, \mathcal{R}_{2}\right\}$, where $R_{1}+R_{2}=0 . \mathcal{R}_{1}: \mathcal{S} \times \mathcal{A} \rightarrow \mathbb{R}$ is the reward function for the defender and $\mathcal{R}_{2}$ is the reward function for the attacker.

The defender goal is to maximize the expected sum of discounted rewards. Under randomized defense and attack policies $\pi$ and $\theta$, the expected sum of discounted game value function starting at an initial state $s_{0} \in \mathcal{S}$ can be expressed as,

$$
V(s, \pi, \theta)=\mathbb{E}\left[\sum_{t=0}^{\infty} \gamma^{t} R\left(s^{t}, a_{1}^{t}, a_{2}^{t}, s^{t+1}\right) \mid s_{0}=s, \pi, \theta\right],
$$

where $\gamma<1$ is a discount factor.

For POSG, the value of a state is hidden and therefore the defender goal is to maximize the value of each belief he has for every state $s \in \mathcal{S}[2,15,19]$. Moreover, for cyber attacks over graphs, it is more practical to consider a finite horizon game model. This is due to the fixed size of the attack graph. Hence, an attacker can reach her targeted node within a finite number of actions. This ensures that the game is finite, and hence the game has at least one equilibrium [4].

\subsection{Defender action}

Let $\hat{\mathcal{G}}$ denote the network before taking any deceptive actions by the defender. Such a network has an associated attack graph, $\mathcal{G}_{a}$ that is generated based on the nodes' host-type of this network as well as its vulnerabilities according to the National Vulnerability Database [26]. One can use MulVAL [27] to automatically generate the attack graph. Since the attacker is uncertain about the true graph of the network, the defender action $a_{1} \in \mathcal{A}_{1}$ strives to increase such uncertainty by adding honeypots to the network attack graph as a fake exploitable vulnerabilities between any two hosts. The defender incurs a cost associated with every action $a_{1}$, denoted as $C_{1}\left(s, a_{1}\right)$

As a result, the network vulnerabilities change and hence the associated attack graph of the network as observed by the attacker. 
This results in a noisy observation (i.e., partial information) for the attacker of the state.

At each state $s \in \mathcal{S}$, the defender action $a_{1}$ is to decide where to place a honeypot (i.e, new edge). Given the associated cost of adding a honeypot, the defender may choose to stay idle. We assume that the defender only places one new honeypot at a time. Therefore, the defender has a maximum of $N(N-1)$ pure actions to consider, where $N=|\mathcal{V}|$ is the number of hosts in the network.

\subsection{Attacker action}

To consider a practical threat model, we assume that the attacker knows that the observed network is deceptive, but on the other hand, the attacker does not know how honeypots are allocated. The attacker goal is to maximize her reward given this partial information. Her goal is to compromise the network via exploiting its known vulnerabilities. To achieve her goal, the attacker first observes the current state of the network partially at time $t, o^{t} \in \mathrm{O}_{2}$, after the defender applied his deceptive action to the network. Therefore, the attacker cannot distinguish between honeypot vulnerabilities and real vulnerabilities. Given the imperfect information collected during the reconnaissance stage, the attacker action $a_{2}$ is to select which vulnerability to exploit next. Due to the fact that the attack graph is directed, the permissible attacker action set at state $s$ is limited to a subset of neighboring nodes of her most recent attacked node.

\subsection{State-space}

In order to capture all of the information needed to describe the network state at every stage, a state $s \in \mathcal{S}$ is a defined as:

$$
s=\left(\mathcal{E}_{s}, \mathcal{I}_{s}, \mathcal{T}_{s}\right),
$$

where $\mathcal{E}_{s}$ is the set of vulnerabilities on the attack graph at state $s$, which is observable to both players. The set $\mathcal{I}_{s}$ denotes the identity set of each vulnerability. Finally, the set $\mathcal{T}_{s}$ is the set of exploited vulnerabilities up to the current stage $s$.

In this formulation we assume that the defender fully observes the set of vulnerabilities $\mathcal{E}_{s}$, as well as the edges' identities , $\mathcal{I}_{s}$. The defender is assumed to have partial observation regarding which vulnerability has been exploited so far. On the other side, the attacker knows exactly What vulnerabilities have been exploited (i.e., $\mathcal{T}_{s}$ ). We assume also that the attacker knows the attack graph structure, however she is uncertain about the identity of each edge.

State transition: The attack graph evolves from state $s$ to state $s^{\prime}$ according to the following equation.

$$
s^{\prime}=f\left(s, a_{1}, a_{2}, \zeta\right),
$$

for some deterministic function $f($.) where $\zeta$ is a random variable that captures the randomness of the network evolution and the associated attack graph.

\subsection{Payoff functions}

Finally, we complete our game model by defining the reward functions. The immediate payoff experienced by both players is a function of the current state $s$ and the joint action played by the players, $\left(a_{1}, a_{2}\right)$. If the attacker decided to exploit a vulnerability $(u, v)$, she incurs a high cost if $\mathcal{I}_{s}(u, v)=h$, where $h$ means a honeypot. In this case the defender gains a reward. On the other hand, the attacker gains a reward if the attacked vulnerability is a true one, i.e, $\mathcal{I}_{s}(u, v) \neq h$. We can readily define the defender reward as:

$$
R_{1}\left(s, a_{1}, a_{2}\right)=\alpha 1_{\left\{I_{s}\left(a_{2}\right)=h\right\}}-\eta 1_{\left\{I_{s}\left(a_{2}\right) \neq h\right\}}-C_{1}\left(s, a_{1}\right)+C_{2}\left(s, a_{2}\right),
$$

where $1_{\{.\}}$is an indicator function, $\alpha$ is the reward received by the defender when the attacker hits a honeypot, and $\eta$ is the loss if a true node is exploited. There is a cost associated with every possible action defined by the functions $C_{1}\left(\right.$.) and $C_{2}($.). The cost function takes into consideration the topological importance of the attacked host. At the same time the location of the honeypot added to the network affects the cost paid by the defender. The attacker immediate payoff function is $R_{2}=-R_{1}$ as we mentioned earlier.

\section{SOLUTION APPROACHES AND DISCUSSION}

\subsection{Solution approaches}

Several techniques have been proposed to solve POSG by extending existing algorithms for single-agent Partially Observable Markov Decision Processes (POMDPs). For example, in [15], the authors proposed an algorithm to solve POSG that synthesizes the dynamic programming operator in POMDP. A POMDP is a single agent POSG. The agent models his/her uncertainty about the state using beliefs. A state belief is a probability distribution defined over the state-space. In other words, the belief space $\mathcal{B}=\Delta(\mathcal{S})$ that consists of all possible beliefs about any current state $s \in \mathcal{S}$. This step converts a POMDP into an MDP defined over the belief space $\mathcal{B}$. The algorithm combines the dynamic programming operator with iterated elimination of dominated strategies. This algorithm has shown to converge to optimal strategies for both players in special cases. Other approaches proposed approximate techniques to enhance solution scalability $[7,22]$. These approaches provide a good starting point for solving our game model, though we expect that they will not be scalable for realistic networks without further improvement and exploiting domain-specific structure due to the complexity of our games.

\subsection{Game complexity}

For the proposed game formulation, each player has his/her own belief space. For instance, the defender belief $b_{1}(s)$ is reduced to $b_{1}\left(\mathcal{T}_{s}\right)$, i.e, a probability associated to the vector of exploited vulnerabilities. Similarly, the attacker state belief $b_{2}(s)=b_{2}\left(\mathcal{I}_{s}\right)$.

This representation reduces the dimensionality of the state-belief space for both players. However, it is still computationally expensive for each player to reason about all the possible states which grows exponentially with the network size. We also note that the players must recursively model the beliefs of the other players in the solution to the POSG. However, the sequential decisions taken by the defender limit the number of pure actions to $N(N-1)$, as the defender decides the location of one honeypot at a time. Also, the attacker selects one vulnerability to attack at a time or decides to back off. This limits the game complexity played at each stage to some extent; it is also likely that realistic versions of this game would be limited to a fairly small number of sequential moves by each player so we would not need to consider very long time horizons. 
Nevertheless, it is obvious that the scalability of existing dynamic programming algorithm can be enhanced through approximate techniques to obtain a more compact representation of the state space $[2,6]$. We plan to investigate these compact representations and other approximation techniques to be able to solve realistic versions of these POSG with two-sided uncertainty in future work.

\section{CONCLUSION}

In this paper, we propose a new formulation that captures the dynamics of cyber deception games between a network defender and an adversary. This formulation considers a practical threat model that accounts for attacker uncertainty about the network state. The reward functions capture important tradeoffs between the defense cost and the loss due to compromising a node. Finally, we discuss the complexity of the game and the feasibility of possible approaches to solve the game. We also provide some techniques to simplify the state space representation to enhance the scalability of existing algorithms.

\section{ACKNOWLEDGMENT}

Research was sponsored by the Army Research Laboratory and was accomplished under Cooperative Agreement Number W911NF-192-0150. The views and conclusions contained in this document are those of the authors and should not be interpreted as representing the official policies, either expressed or implied, of the Army Research Laboratory or the U.S. Government. The U.S. Government is authorized to reproduce and distribute reprints for Government purposes notwithstanding any copyright notation herein.

\section{REFERENCES}

[1] Bo An, James Pita, Eric Shieh, Milind Tambe, Chris Kiekintveld, and Janusz Marecki. 2011. GUARDS and PROTECT: Next generation applications of security games. ACM SIGecom Exchanges 10, 1 (2011), 31-34.

[2] Ahmed H Anwar, George Atia, and Mina Guirguis. 2017. Dynamic game-theoretic defense approach against stealthy Jamming attacks in wireless networks. In Communication, Control, and Computing (Allerton), 2017 55th Annual Allerton Conference on. IEEE, 252-258.

[3] DEPARTMENT OF THE ARMY. 2019. Army Support to Military Deception. (2019). https://fas.org/irp/doddir/army/fm3-13-4.pdf

[4] Tamer Başar and Geert Jan Olsder. 1998. Dynamic noncooperative game theory. SIAM

[5] Mark Bilinski, Ryan Gabrys, and Justin Mauger. 2018. Optimal Placement of Honeypots for Network Defense. In International Conference on Decision and Game Theory for Security. Springer, 115-126.

[6] Branislav Bosansky, Albert Xin Jiang, Milind Tambe, and Christopher Kiekintveld 2015. Combining compact representation and incremental generation in large games with sequential strategies. In Twenty-Ninth AAAI Conference on Artificial Intelligence.

[7] Lucian Bu, Robert Babu, Bart De Schutter, et al. 2008. A comprehensive survey of multiagent reinforcement learning. IEEE Transactions on Systems, Man, and Cybernetics, Part C (Applications and Reviews) 38, 2 (2008), 156-172.

[8] Thomas E Carroll and Daniel Grosu. 2011. A game theoretic investigation of deception in network security. Security and Communication Networks 4, 10 (2011), $1162-1172$.

[9] Hayreddin Çeker, Jun Zhuang, Shambhu Upadhyaya, Quang Duy La, and BoonHee Soong. 2016. Deception-based game theoretical approach to mitigate DoS attacks. In International Conference on Decision and Game Theory for Security. Springer, $18-38$

[10] Andrew Clark, Quanyan Zhu, Radha Poovendran, and Tamer Başar. 2012. Deceptive routing in relay networks. In International Conference on Decision and Game Theory for Security. Springer, 171-185.

[11] Karel Durkota, Viliam Lisỳ, Branislav Bošanskỳ, and Christopher Kiekintveld. 2015. Approximate solutions for attack graph games with imperfect information. In International Conference on Decision and Game Theory for Security. Springer, 228-249.
[12] Karel Durkota, Viliam Lisỳ, Branislav Bosanskỳ, and Christopher Kiekintveld. 2015. Optimal Network Security Hardening Using Attack Graph Games.. In IFCAI. 526-532.

[13] Karel Durkota, Viliam Lisỳ, Christopher Kiekintveld, Branislav Bošanskỳ, and Michal Pěchouček. 2016. Case studies of network defense with attack graph games. IEEE Intelligent Systems 31, 5 (2016), 24-30.

[14] Patrick Engebretson. 2013. The basics of hacking and penetration testing: ethical hacking and penetration testing made easy. Elsevier.

[15] Eric A Hansen, Daniel S Bernstein, and Shlomo Zilberstein. 2004. Dynamic programming for partially observable stochastic games. In $A A A I$, Vol. 4. 709715.

[16] Cisco Visual Networking Index. 2017. Global mobile data traffic forecast update, 2016-2021 white paper. Cisco: San fose, CA, USA (2017).

[17] Manish Jain, Dmytro Korzhyk, Ondřej Vaněk, Vincent Conitzer, Michal Pěchouček, and Milind Tambe. 2011. A double oracle algorithm for zero-sum security games on graphs. In The 10th International Conference on Autonomous Agents and Multiagent Systems-Volume 1. 327-334.

[18] Sushil Jajodia, Paulo Shakarian, VS Subrahmanian, Vipin Swarup, and Cliff Wang. 2015. Cyber warfare: building the scientific foundation. Vol. 56. Springer.

[19] Leslie Pack Kaelbling, Michael L Littman, and Anthony R Cassandra. 1998. Planning and acting in partially observable stochastic domains. Artificial intelligence 101, 1-2 (1998), 99-134.

[20] Charles A Kamhoua. 2018. Game theoretic modeling of cyber deception in the Internet of Battlefield Things. In 2018 56th Annual Allerton Conference on Communication, Control, and Computing (Allerton). IEEE, 862-862.

[21] Alexander Kott, Ananthram Swami, and Bruce J West. 2016. The internet of battle things. Computer 49, 12 (2016), 70-75.

[22] Akshat Kumar and Shlomo Zilberstein. 2009. Dynamic programming approximations for partially observable stochastic games. In Twenty-Second International FLAIRS Conference.

[23] Joshua Letchford and Yevgeniy Vorobeychik. 2013. Optimal interdiction of attack plans. In Proceedings of the 2013 international conference on Autonomous agents and multi-agent systems. International Foundation for Autonomous Agents and Multiagent Systems, 199-206.

[24] Yapeng Li, Yu Xiao, Yong Li, and Jun Wu. 2018. Which Targets to Protect in Critical Infrastructures-A Game-Theoretic Solution From a Network Science Perspective. IEEE Access 6 (2018), 56214-56221.

[25] Gordon Fyodor Lyon. 2009. Nmap network scanning: The official Nmap project guide to network discovery and security scanning. Insecure.

[26] National Vulnerability Database . [n.d.]. https://nvd.nist.gov/. https://nvd.nist.gov/

[27] Xinming Ou, Wayne F Boyer, and Miles A McQueen. 2006. A scalable approach to attack graph generation. In Proceedings of the 13th ACM conference on Computer and communications security. ACM, 336-345.

[28] Xinming Ou, Sudhakar Govindavajhala, and Andrew W Appel. 2005. MulVAL: A Logic-based Network Security Analyzer.. In USENIX Security Symposium, Vol. 8. Baltimore, MD

[29] Radek Píbil, Viliam Lisỳ, Christopher Kiekintveld, Branislav Bošanskỳ, and Michal Pěchouček. 2012. Game theoretic model of strategic honeypot selection in computer networks. In International Conference on Decision and Game Theory for Security. Springer, 201-220.

[30] James Pita, Manish Jain, Janusz Marecki, Fernando Ordóñez, Christopher Portway, Milind Tambe, Craig Western, Praveen Paruchuri, and Sarit Kraus. 2008. Deployed ARMOR protection: the application of a game theoretic model for security at the Los Angeles International Airport. In Proceedings of the 7th international joint conference on Autonomous agents and multiagent systems: industrial track. International Foundation for Autonomous Agents and Multiagent Systems, 125132

[31] Neil C Rowe and Han C Goh. 2007. Thwarting cyber-attack reconnaissance with inconsistency and deception. In Information Assurance and Security Workshop, 2007. IAW'07. IEEE SMC. IEEE, 151-158.

[32] Aaron Schlenker, Omkar Thakoor, Haifeng Xu, Fei Fang, Milind Tambe, Long Tran-Thanh, Phebe Vayanos, and Yevgeniy Vorobeychik. 2018. Deceiving cyber adversaries: A game theoretic approach. In Proceedings of the 17th International Conference on Autonomous Agents and MultiAgent Systems. International Foundation for Autonomous Agents and Multiagent Systems, 892-900.

[33] Jason Tsai, Christopher Kiekintveld, Fernando Ordonez, Milind Tambe, and Shyamsunder Rathi. 2009. IRIS-a tool for strategic security allocation in transportation networks. (2009).

[34] Yevgeniy Vorobeychik. 2013. Optimal interdiction of attack plans. Technical Report. Sandia National Laboratories.

[35] Wenyuan Xu, Wade Trappe, Yanyong Zhang, and Timothy Wood. 2005. The feasibility of launching and detecting jamming attacks in wireless networks. In Proceedings of the 6th ACM international symposium on Mobile ad hoc networking and computing. ACM, 46-57.

[36] Tao Zhang and Quanyan Zhu. 2018. Hypothesis Testing Game for Cyber Deception. In International Conference on Decision and Game Theory for Security. Springer, 540-555. 